Intensivmed 2011 • 48:6-6

DOI 10.1007/s00390-011-0260-3

Online publiziert: 2. Februar 2011

(c) Springer-Verlag 2011

\section{Gortner}

Klinik für Allgemeine Pädiatrie und Neonatologie, Kliniken für Kinder- und Jugendmedizin, Universitätsklinikum des Saarlandes, Homburg/Saar

\title{
Der pädiatrische Notfall
}

Das Spektrum der Notfälle in der Kinderund Jugendmedizin hat sich während der vergangenen zwei Dekaden bedeutsam gewandelt. Bedingt durch die verbesserte prästationäre Diagnostik und Therapie wurden Kinder mit obstruktiven Atemwegserkrankungen, wie z. B. Krupp-Syndrom und Status asthmaticus, in diesem Zeitraum immer seltener stationär aufgenommen. Dem gegenüber nahmen in der Jugendmedizin Intoxikationen, wie z. B. durch Alkohol und Drogen, um den Faktor 3 bis $4 \mathrm{zu}$. Jugendliche und Erwachsene mit angeborenen Herzfehlern und Operation im Neugeborenen- und Säuglingsalter sind in der Notfall- und Intensivmedizin eine wachsende Patientengruppe. Diese Gruppe ist z. B. nach Operationen mittels Vorhofumkehr bei Transposition der großen Gefäße, hochgradig gefährdet durch Rhythmusstörungen, die einen bedrohlichen Verlauf annehmen können. Diese Patienten werden aufgrund der erst seit etwa 20 Jahren bestehenden Behandlungsmöglichkeiten zunehmend häufiger beobachtet. Dasselbe gilt für Jugendliche und junge Erwachsene mit Herzinsuffizienz bei schweren kritischen Vitien mit Operationen im frühen Kindesalter. Um die richtigen diagnostischen und therapeutischen Schritte schon im notfallmedizinischen Sektor einleiten zu können, sind eine sorgfältige Kenntnis der zugrundeliegenden angeborenen Herzfehler, ihrer operativen Versorgung am Beginn des Lebens und deren mittelfristige Konsequenzen in hämodynamischer Sicht bis in das adoleszente und frühe Erwachsenenalter hinein notwendig. Dieser Problematik sind zwei Artikel zu den Aspekten Herzinsuffizienz und bedrohliche Herzrhythmusstörungen gewidmet.

Weiterhin haben sich die Möglichkeiten der apparativen, auch extrakorporalen, Therapie im Kindesalter beim Lungenversagen während der letzten Dekade enorm ausgeweitet. Die Therapie mittels der extrakorporalen Membranoxygenierung (ECMO) ist nicht nur für die neonatologische Intensivmedizin bei Vorliegen angeborener Zwerchfellhernien, eines Mekoniumaspirationssyndroms und anderen Erkrankungen mit schwerem respiratorischem Versagen, sondern auch bei älteren Kindern mit ARDS zunehmend Standard.

Bei der respiratorischen Insuffizienz des Neugeborenen wurden neue Beatmungsverfahren entwickelt, die weniger invasiv sind, z. B. mittels nichtinvasiver Beatmung durch erweiterten Gebrauch des kontinuierlich positiven Atemwegsdrucks (CPAP). Das Verfahren wird jetzt auch bei älteren Kindern über Larynxmaske eingesetzt. Weiterhin ist die Hochfrequenzoszillationsbeatmung sowohl in der Neonatologie beim Versagen der konventionellen Beatmung sowie bei schweren therapierefraktären Fällen mit ARDS vor Einsatz einer ECMO zunehmend Standard in der pädiatrischen Intensivmedizin. Dieser Problemkreis wird in zwei Artikeln aufgegriffen und die Definition von Therapiestandards in der neonatologischen und pädiatrischen Intensivmedizin dargestellt.

\section{(D) Die Hypothermietherapie hat sich als Standard bei neonataler Asphyxie etabliert}

Daneben wird das ewig junge Thema der Notfälle beim Neugeborenen angesprochen. Die Implementierung neuer internationaler Leitlinien, wie z. B. zum Mekoniumaspirationssyndrom, sind höchst umstritten und werden daher in einem Beitrag aufgegriffen: Das Unterlassen der Absaugung der Atemwege bei Spontangeburt nach Durchtritt des Kopfes hat in den entwickelten Ländern zu einer drastischen Rate der schweren Mekoniumaspirationssyndrome geführt und wird hier nicht mehr empfohlen. Basis für die neuen Empfehlungen, u. a. des ILCOR (International Liaison Committee on Resuscitation), ist eine einzige Studie, die zum Teil in Schwellenländern durchgeführt wur- de, und deren Aussage daher nicht unkommentiert auf die Therapiestandards in unserem Versorgungsbereich übertragen werden darf.

Weiterhin sind Störungen der respiratorischen Adaptation bei angeborenen oder unter Geburt erworbenen Störungen der Lungenfunktion Gegenstand der Diskussion in diesem Beitrag, der daneben auf eine neue Therapieoption in der Neonatologie bei mittelschwerer und schwerer Asphyxie verweist. Die Hypothermietherapie mit rund $34^{\circ} \mathrm{C}$ über 48 Stunden hat sich mittlerweile als Therapiestandard auch leitlinienbasiert etabliert und stellt nach zwei Dekaden klinisch weitgehend irrelevanter Forschungsresultate einen Meilenstein in der Behandlung bei neonataler Asphyxie dar.

Gerade die Problematik der Patienten mit angeborenen Herzfehlern im Jugendlichen- und Erwachsenenalter zeigt, dass die enge Interaktion zwischen den verschiedenen intensivmedizinischen Disziplinen unabdingbar ist, um weitere Fortschritte zu realisieren und mit den veränderten Patientencharakteristika, mit denen wir uns konfrontiert sehen, weiter auf höchstem Niveau umgehen zu können.
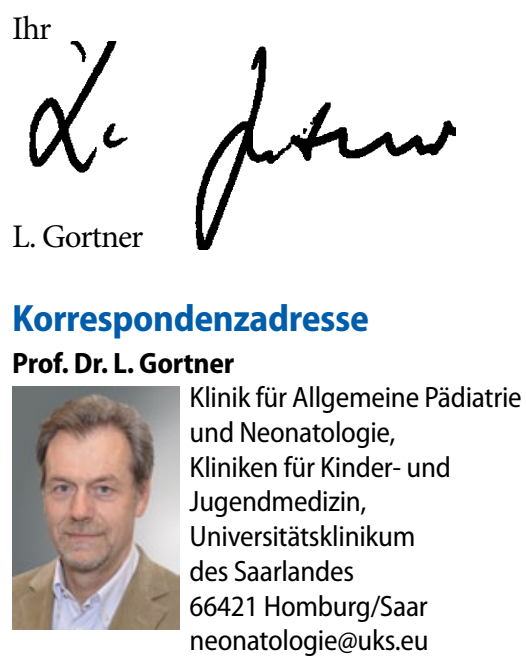